\title{
Paxillin is positively correlated with the clinicopathological factors of colorectal cancer, and knockdown of Paxillin improves sensitivity to cetuximab in colorectal cancer cells
}

\author{
CHUANG DU ${ }^{1}$, XIN WANG $^{1}$, JUNLING ZHANG ${ }^{1}$, XIANGZHENG LIU ${ }^{2}$, JING ZHU $^{1}$ and YUCUN LIU ${ }^{1}$ \\ Departments of ${ }^{1}$ General Surgery and ${ }^{2}$ Thoracic Surgery, Peking University First Hospital, Beijing 100034, P.R. China
}

Received March 22, 2015; Accepted September 18, 2015

DOI: $10.3892 /$ or.2015.4352

\begin{abstract}
Paxillin (PXN) encodes a $68-\mathrm{kDa}$ focal adhesionassociated protein and plays an important role in signal transduction, regulation of cell morphology, migration, proliferation and apoptosis. The aim of the present study was to evaluate the relationship between PXN and clinicopathological factors in colorectal cancer, the role of PXN in cetuximab resistance, and whether knockdown of PXN expression could improve the sensitivity to cetuximab in colorectal cancer cells. In the present study, immunohistochemical staining in 148 colorectal carcinoma and 126 normal adjacent tissues was performed, which showed that the positive rate of PXN was significantly higher in the colorectal adenocarcinoma samples than that in the normal colorectal mucosa samples $(\mathrm{P}<0.001)$. Moreover, PXN presence was also positively correlated with TNM stage $(\mathrm{P}=0.023)$, distant metastasis $(\mathrm{P}=0.014)$, recurrence $(\mathrm{P}=0.032)$ and reduced survival $(\mathrm{P}=0.004)$. In vitro, $\mathrm{PXN}$ expression was positively correlated with the proliferation rate in colorectal cells insensitive to cetuximab. Inhibition of PXN expression by PXN-siRNA clearly increased apoptosis by downregulating the phosphorylation of extracellular signal regulated kinase (p-Erk) level, and overexpression of PXN by PXN-cDNA decreased apoptosis by upregulating the p-Erk level. This suggests that overexpression of PXN could be one of the reasons for cetuximab resistance, and downregulation of PXN plays an important role in improving sensitivity to cetuximab by suppressing the activitation of p-Erk in colorectal cancer cells. Above all, knockdown of PXN could represent a rational therapeutic strategy for increasing the sensitivity or overcoming cetuximab-resistance in patients with colorectal cancer.
\end{abstract}

Correspondence to: Professor Xin Wang, Department of General Surgery, Peking University First Hospital, 8 Xishiku Street, Xicheng, Beijing 100034, P.R. China

E-mail: wangxin_guo@126.com

Key words: paxillin, colorectal cancer, p-Erk, apoptosis, cetuximab resistance, clinicopathological factors

\section{Introduction}

Colorectal cancer is one of the most frequently diagnosed malignant diseases and one of the leading causes of cancer-related death worldwide (1). In China, the incidence of colorectal cancer increases by $\sim 4.2 \%$ year, and there is a higher incidence among younger adults (2). Moreover, metastatic cancer occurs in 40-50\% of newly diagnosed patients, which is associated with high morbidity (3). Recent therapeutic strategies for metastatic colorectal cancer (mCRC) have focused on developing molecular-targeted therapies.

It has been reported that increased epidermal growth factor receptor (EGFR) expression is the hallmark of many human tumors and an important therapeutic target in mCRC (4). Cetuximab, the first chimeric monoclonal antibody which has been generated against the EGFR, is the first-line treatment used as a single agent or in combination with standard chemotherapy for KRAS wild-type mCRC patients (5). The mechanism of cetuximab (C225, Erbitux) involves the specific binding of the extracellular domain of EGFR, subsequently blocking the downstream signaling of EGFR that influences cell proliferation, survival, apoptosis, migration and tumorigenesis. Numerous clinical studies have shown that cetuximab significantly improves the progression-free and overall survival of patients with KRAS wild-type mCRC, while patients with mutant KRAS do not benefit from cetuximab treatment $(6,7)$. The main reason for this phenomenon is that downstream signaling of KRAS in the MAPK pathway is not controlled by EGFR. Moreover, KRAS gene mutations, BRAF, NRAS and PIK3CA gene mutations can also cause resistance to cetuximab in mCRC patients $(6,8)$. However, the resistance to cetuximab still exits in patients with the wild-type genes mentioned above, which indicates that there are some other resistance mechanisms to be explored (9).

Several studies have shown that phosphorylation levels of paxillin $(\mathrm{PXN})$ are related to those of cytokine receptors; the blocking of cell growth factor receptors leads to a compensatory increase in PXN phosphorylation levels, and that PXN plays an important role in mediating signal transduction of the epidermal growth factor (EGF) (2,10-12). Therefore, high expression of PXN and increased phosphorylation levels may be one of the reasons for the poor therapeutic effect of cetuximab. Meanwhile, it is necessary and meaningful to explore the specific mechanism of resistance to cetuximab in mCRC. 
PXN, a 68-kDa focal adhesion-associated protein, contains a number of motifs that mediate protein-protein interactions, including C-terminal LIM domains resembling a double zinc-finger domain, $\mathrm{N}$-terminal LD motifs, $\mathrm{SH} 3$ and $\mathrm{SH} 2$ domain-binding sites, whose motifs serve as docking sites for cytoskeletal proteins, tyrosine and serine/threonine kinases, GTPase-activating proteins and other adaptor proteins $(13,14)$. PXN plays an important role in signal transduction, regulation of cell morphology, migration, proliferation and apoptosis $(2,13,14)$. A number of studies have demonstrated that high expression of PXN also occurs in cancer cells of many other organs, such as the esophagus, prostate, and lung (2). Upregulation of PXN phosphorylation levels promotes tumor cell growth, invasion, migration, recurrence and inhibits cell apoptosis, which results in the poor prognosis of patients. However, expression of PXN does not serve as an independent risk factor for prognosis $(15,16)$. Various studies have mentioned that PXN mediates the EGF signal transduction process. Sen et al discovered that after knockdown of the PXN gene in prostate cancer cells, the phosphorylation level of extracellular regulated-protein kinase (Erk) significantly decreased in the downstream of mitogen-activated protein kinase (MAPK) pathway, which proves that activation of the Erk signaling pathway requires PXN involvement and PXN is necessary for the proliferation of prostate cancer cells (11). In a study of non-small cell lung cancer (NSCLC), Wu et al found that activation of Erk requires mediation of PXN and further confirms that expression of the B-cell leukemia/lymphoma-2 (Bcl-2) gene is regulated by PXN through activation of Erk, while high expression of the $\mathrm{Bcl}-2$ gene inhibits cancer cell apoptosis, causing resistance to chemotherapeutic drugs in lung cancer patients (17). Despite these previous studies, whether PXN overexpression is one of the reasons for resistance to cetuximab and how PXN affects the MAPK pathway in colorectal cancer cells remain unknown.

In the present study, immunohistochemical staining (IHC) in 148 colorectal carcinoma and 126 normal adjacent tissues was performed to clarify the relationship between TNM stage, recurrence rate, metastasis, survival and PXN expression. Two human colon cancer cell lines were chosen to discover the role of PXN activation in cetuximab resistance: SW480 cells with high expression of PXN and insensitive to cetuximab, and Caco-2 cells with the opposite characteristics. It was demonstrated that inhibition of PXN expression by PXN-siRNA restored sensitivity to cetuximab in the SW480 cells, and upregulation of PXN expression by PXN-cDNA (PXN Human cDNA ORF Clone) reduced the sensitivity to cetuximab in the Caco-2 cells. Notably, the expression of p-Erk and PXN expression were consistent. We treated these cells with a selective Erk inhibitor, which restored cetuximab sensitivity in the SW480 cells and made Caco- 2 cells more sensitive to cetuximab. These results suggest that inhibition of PXN expression can improve sensitivity to cetuximab by downregulation of p-Erk levels.

\section{Materials and methods}

Drugs. Cetuximab, an anti-EGFR human-mouse chimeric monoclonal antibody $(\mathrm{mAb})$, was purchased from Merck Serono (Darmstadt, Germany). SCH-772984, a selective inhibitor of extracellular signal regulated kinase (Erk1/2) that displays behaviors of both type I and II kinase inhibitors, was purchased from Selleckchem. SCH-772984 was dissolved in dimethylsulfoxide (DMSO) and maintained as a concentrated stock at $-20^{\circ} \mathrm{C}$. Working concentrations were diluted in culture medium just before each experiment.

Tumor samples. We received approval for the present study from the Clinical Research Ethics Committee, and tumor specimens were collected after obtaining patient informed consent in accordance with the institutional guidelines. We selected 274 samples from 148 patients (78 men and 70 women) with primary colorectal carcinoma who underwent surgery at the Department of General Surgery, Peking University First Hospital from January 2006 to June 2009, including 148 colorectal carcinoma and 126 normal adjacent mucosa (at least $2 \mathrm{~cm}$ distant from the tumor). The patients were selected according to TNM stage; the case number of each stage did not differ greatly. None of the patients had received chemotherapy and radiotherapy before surgery. Two pathologists reviewed all of the histological slides.

Cell lines. The human colon cancer cell lines and a human colon normal mucosa cell line were obtained from the Colon Cancer Institute of Peking University First Hospital (Beijing, China), including SW480, HT29, Rko, Caco-2, Lovo and FHC cell lines. These cells were routinely cultured in Dulbecco's modified Eagle's medium [DMEM containing 10\% fetal bovine serum (FBS)] (both from Gibco) and penicillin/streptomycin at $37^{\circ} \mathrm{C}$ in a $5 \% \mathrm{CO}_{2}$-humidified atmosphere.

Proliferation assay. Cancer cells were seeded into 96-well plates and treated with different concentrations of SCH-772984 (range, $5-90 \mathrm{mg} / \mathrm{ml}$ ), cetuximab (range $5-700 \mathrm{mg} / \mathrm{ml}$ ) alone for $48 \mathrm{~h}$ or with cetuximab $(100 \mathrm{mg} / \mathrm{ml})$ for a period of time (6 h-10 days). Cell proliferation was measured with MTT assay. The $\mathrm{IC}_{50}$ value and proliferation rate were calculated according to optical density from a microplate reader. Each test was performed in quadruplicate.

Apoptosis assay. SW480 and Caco-2 cells were seeded in $25 \mathrm{~cm}^{2}$ flasks, treated for $72 \mathrm{~h}$ and stained with Annexin V-fluorescein isothiocyanate (FITC) and 7-aminoactinomycin D (7-AAD) in the dark. Viable (7-AAD-negative) and dead (7-AAD-positive) cell populations were quantitated by flow cytometry using a flow cytometer (BD Influx).

RNA interference. The small inhibitor duplex RNAs (siRNAs) (On-target paxillin) PXN-siRNAs (human: SR303929) were purchased from OriGene. OriGene synthesized three siRNA duplexes targeting human paxillin mRNA (PXN-siRNA). The targeting sequences were siRNA1, UGACGAAAGAGAAG CCUAAGCGGAA; siRNA2, UGAACGCUGUACAGCAU AACCCGCC; and siRNA3, GACAAUGCCAGCAUAAAUC CAUCCA. In the present study, siRNA1 was used since it effectively inhibited PXN expression in our preliminary experiments. The siCONTROL non-targeting siRNA (human, SR30004) was used as a negative control (NC). Cells were transfected with $40 \mathrm{nmol} / 1$ siRNAs using Lipofectamine 2000 reagent (Invitrogen) according to the manufacturer's 
instructions. The day before the transfection, the cells were plated in $25 \mathrm{~cm}^{2}$ flasks at $50-70 \%$ confluency in medium supplemented with 5\% FBS. Cells were harvested $72 \mathrm{~h}$ after the transfection. Efficiency of gene silencing was detected by western blotting.

PXN-cDNA transient transfection and co-transfection of $P X N-c D N A$ and PXN-siRNA. The human PXN-cDNA was purchased from OriGene (RC213811). It was cloned into vector Pcmv6-Entry (cat. \#PS1000-001) and was verified by full sequencing (NM_002859). This human PXN cDNA was derived from single colony $E$. coli cultures and purified through the OriGene ion-exchange plasmid purification system (PowerPrep. HP Midiprep kits with Prefilters NP 100024). The non-targeting plasmid was used as a negative control. The cells were transfected with $1.5 \mathrm{mg} / \mathrm{ml}$ cDNAs using Lipofectamine 2000 reagent following the manufacturer's protocols. The day before the transfection, the cells were plated in $25 \mathrm{~cm}^{2}$ flasks at $50-70 \%$ confluency in medium supplemented with $10 \%$ FBS. They were harvested $72 \mathrm{~h}$ after the transfection. Efficiency of gene silencing was detected by western blotting. When transfecting PXN-cDNA and PXN-siRNA at the same time, Lipofectamine 2000 reagent and $30 \mathrm{pmol}$ of siRNA/1 mg of DNA was used.

Western blotting. SW480, SW620, HT29, Rko, Caco-2 and Lovo cells were seeded into $25 \mathrm{~cm}^{2}$ flasks and treated for $72 \mathrm{~h}$. Total proteins were obtained by a protein extraction kit (KeyGen Biotech, Nanjing, China), including phosphorylated proteins. The protein concentration was estimated by a modified Bradford assay (KeyGen Biotech). Immunoreactive bands were visualized by enhanced chemiluminescence (ECL; GE Healthcare/Amersham). Antibodies against p-Erk, cleaved caspase-3 and GAPDH (HRP-conjugated) were purchased from Cell Signaling Technology. Antibodies against PXN (Tyr-118) were purchased from Abcam (Hong Kong, China). Secondary antibodies coupled to horseradish peroxidase were purchased from Signalway Antibody (USA). The antibodies were diluted according to the instructions before the experiment. Each experiment was carried out for several times.

$I H C$. The immunohistochemical staining was performed on paraffin sections $(4-\mu \mathrm{m})$ using a streptavidin-peroxidase immunostaining kit. After deparaffinization with xylene and rehydration through a graded ethanol series, the sections were subjected to microwave antigen retrieval with an ethylenediaminetetraacetic acid (EDTA) buffer solution (1 mmol/l, $\mathrm{pH} 8.0$ ) at $98^{\circ} \mathrm{C}$ for $10 \mathrm{~min}$ to unmask antigenic epitopes. Endogenous peroxidase activity was blocked with $0.3 \%$ hydrogen peroxide for $15 \mathrm{~min}$ at room temperature. After blocking non-specific binding sites with a blocking serum for $30 \mathrm{~min}$ at room temperature, the sections were incubated with a mouse monoclonal anti-PXN antibody (1:100; Abcam) at $4^{\circ} \mathrm{C}$ overnight. Visualization of the antibody-enzyme complex was achieved with 3,3-diaminobenzidine tetrahydrochloride (DAB). The sections were counterstained with Mayer's hematoxylin. Appropriate positive controls were included, and as a negative control, normal serum and PBS were substituted for the primary antibody.
Table I. Patient clinicopathological factors and paxillin expression in normal and colorectal cancer tissues.

\begin{tabular}{lrrrr}
\hline & \multicolumn{2}{c}{ Paxillin } & \\
\cline { 3 - 4 } Parameters & Total & + & - & P-value \\
\hline Tissues & & & & $<0.001$ \\
$\quad$ Normal colorectal & 126 & 56 & 70 & \\
mucosa & & & & \\
Colorectal cancer & 148 & 101 & 47 & \\
Distant metastasis & & & & 0.014 \\
Absent & 123 & 79 & 44 & \\
Present & 25 & 22 & 3 & \\
Recurrence & & & & 0.032 \\
Absent & 114 & 73 & 41 & \\
Present & 34 & 28 & 6 & \\
TNM stage & & & & 0.023 \\
I & 28 & 14 & 14 & \\
II & 39 & 24 & 15 & \\
III & 59 & 44 & 15 & \\
IV & 22 & 19 & 3 & \\
\hline
\end{tabular}

Immunohistochemical analysis. To quantify PXN protein expression, both the extent and intensity of immunoreactivity were assessed and scored. In the present study, the scores of the extent of immunoreactivity ranged from 0 to 3 and were determined according to the percentage of cells that exhibited positive staining in each microscopic field of view $(0,<25 \%$; $1,25-50 \% ; 2,>50-75 \%$; and $3,>75-100 \%)$. The scores of IHC intensity were as follows: 0 , negative staining; 1 , weak staining; 2, moderate staining; and 3, strong staining. By multiplying the scores for extent and intensity, a total score ranging from 0 to 9 was achieved. The expression level of PXN was considered high when the total score was $\geq 4$ and low when the total score was $<4$. The results were assessed by two independent observers who did not have any knowledge of the clinicopathological features of the patients. If the results differed, then they consulted and reached a final consensus.

Statistical analysis. The statistical analyses of data were carried out using SPSS 17.0 statistical software (SPSS, Inc., USA). The relationship between PXN expression and clinicopathological characteristics was analyzed by the $\chi^{2}$ test. All $\mathrm{P}$-values represent two-sided tests of statistical significance with P-value $<0.05$.

\section{Results}

The expression of $P X N$ is higher in tumor tissues than that in normal mucosa. The expression of PXN was examined in 148 colorectal adenocarcinoma samples and 126 normal adjacent mucosa samples through an immunohistochemical staining approach. A total of 101 of the 148 (68.2\%) colorectal adonocarcinoma samples and 56 of the 126 (44.4\%) normal tissues were PXN-positive $(\mathrm{P}<0.001)$ (Table I). PXN protein was mainly expressed in the cytoplasm and was found to be 

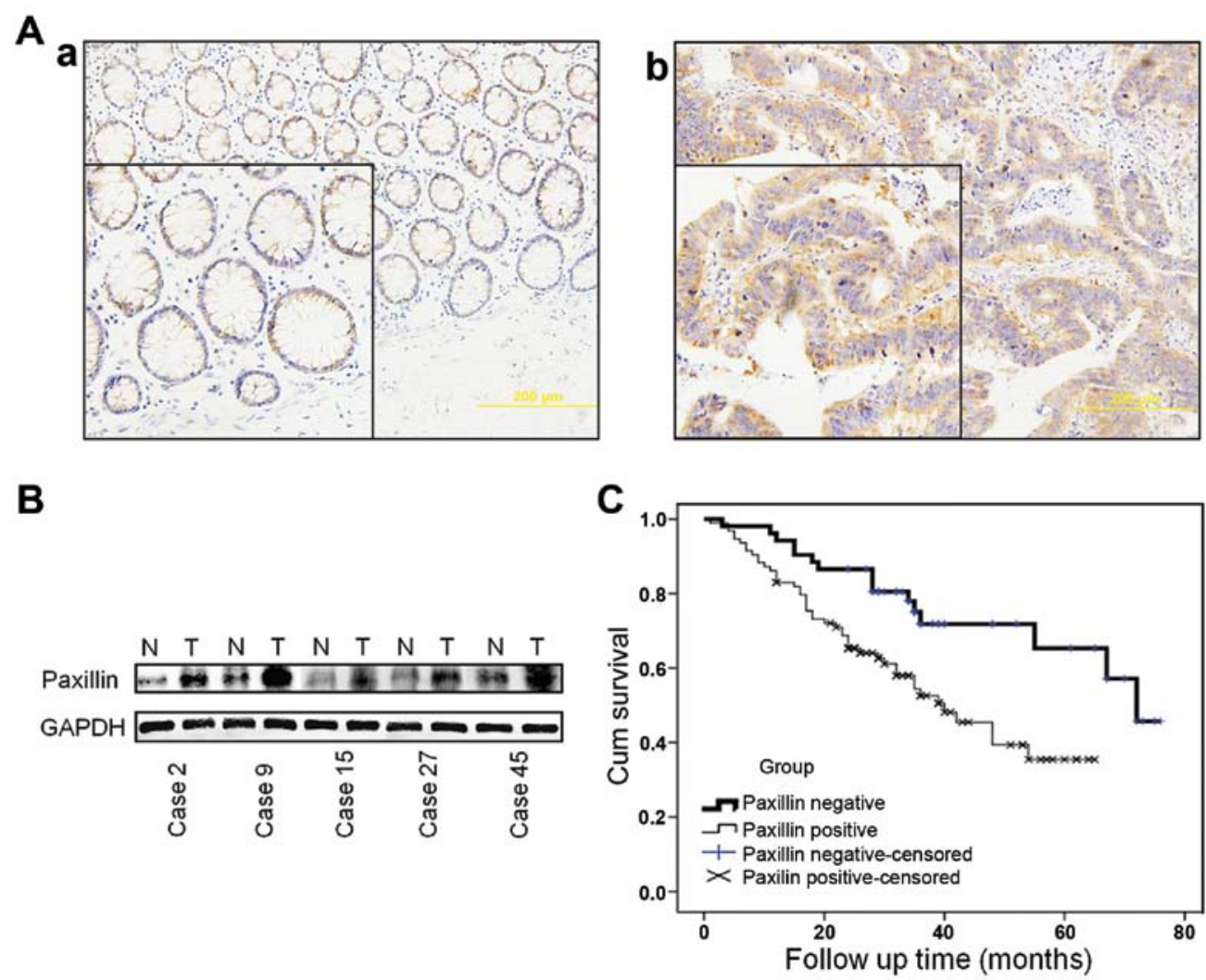

Figure 1. Expression of paxillin is higher in the colorectal tumor tissues than that in the normal mucosa and is negatively related with the survival of patients. (A) Paxillin expression in normal mucosa and colorectal adenocarcinoma (immunohistochemistry) (original magnification, $x 100$ ). a, negative paxillin expression in normal colorectal mucosa; b, positive paxillin expression in colorectal adenocarcinoma. (B) In 43 of 60 (71.7\%) cases, paxillin expression levels in tumors $(\mathrm{T})$ were at least 2 -fold higher than those in normal adjacent mucosa $(\mathrm{N})$. $(\mathrm{C})$ The overall survival of patients in the paxillin-negative group was longer than that in the paxillin-positive group.

overexpressed in the tumors compared with the normal adjacent tissues (Fig. 1A). This result was further confirmed in 60 colorectal carcinoma samples with paired normal adjacent tissues by western blotting. In 43 of 60 (71.7\%) cases, PXN expression levels in the tumor tissues ( $\mathrm{T}$ ) were at least 2-fold higher than levels in the normal adjacent mucosa (N) (Fig. 1B).

PXN expression is negatively correlated with the survival of the patients. Follow-up results in a total of 148 patients were analyzed. The median duration of follow-up was 48.6 months (range 1-75 months), and a total of 63 patients died during the follow-up period. Univariate analyses revealed that the 3- and 5-year survival rates of the PXN-negative group were higher (71.8 and 65.3\%) than those of the positive group (52.5 and $35.4 \%$, log-rank testing, $\mathrm{P}=0.004$, Fig. 1C). The survival rate was correlated with the expression of PXN.

PXN is expressed differently in the colon cell lines. The expression of PXN was assessed in five different colon cancer cell lines and a normal mucosal cell line by western blotting. Compared with FHC, a normal human colon cell line, the 5 colon cancer cell lines showed a higher level of PXN. Among the Caco-2, Lovo, SW480, HT-29 and Rko cells, PXN was expressed at the highest level in the SW480 cells, secondly in the Lovo, HT-29 and Rko cells, and least in the Caco-2 cells (Fig. 2). Therefore, we chose SW480 and Caco-2 cells to perform subsequent experiments.
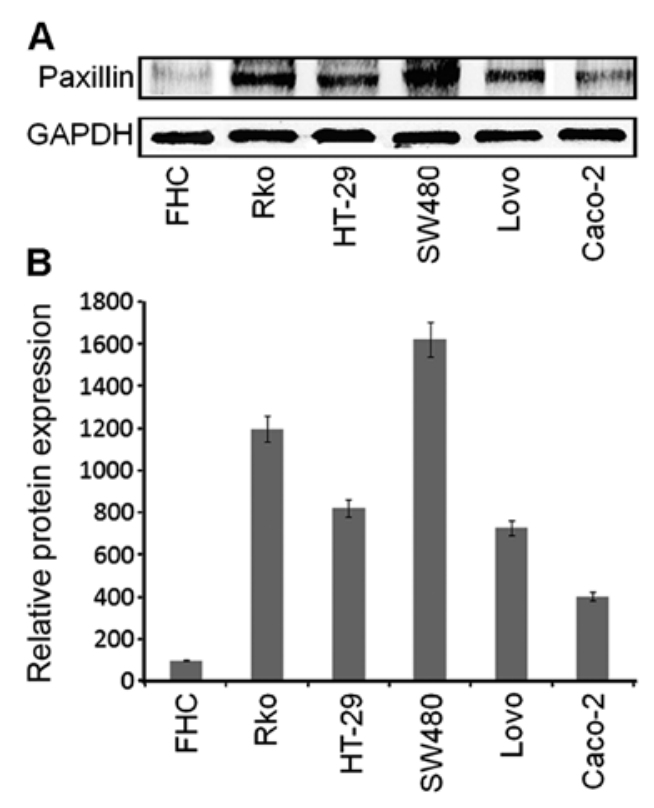

Figure 2. Expression of paxillin differs in the different cell lines. Compared with FHC, a normal human colorectal cell line, 5 colon cancer cell lines showed a higher level of paxillin. The expression of paxillin was the highest in the SW480 cell line and the least in the Caco-2 cell line.

PXN expression in the tumors is related with clinicopathological parameters. The positive rate of PXN was higher in 
A

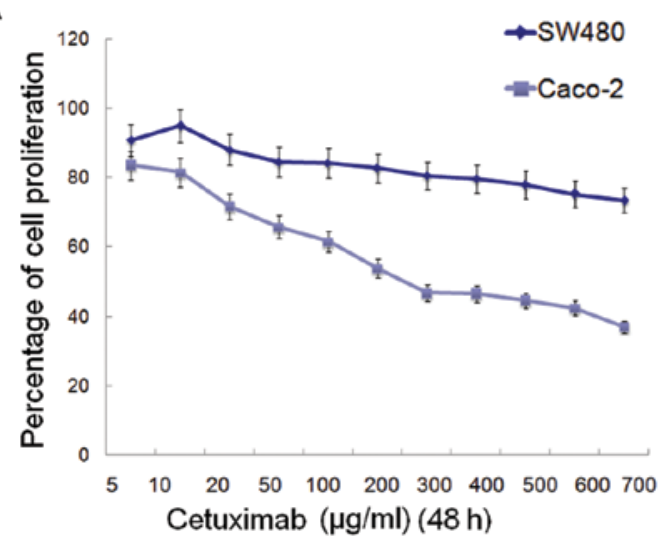

C

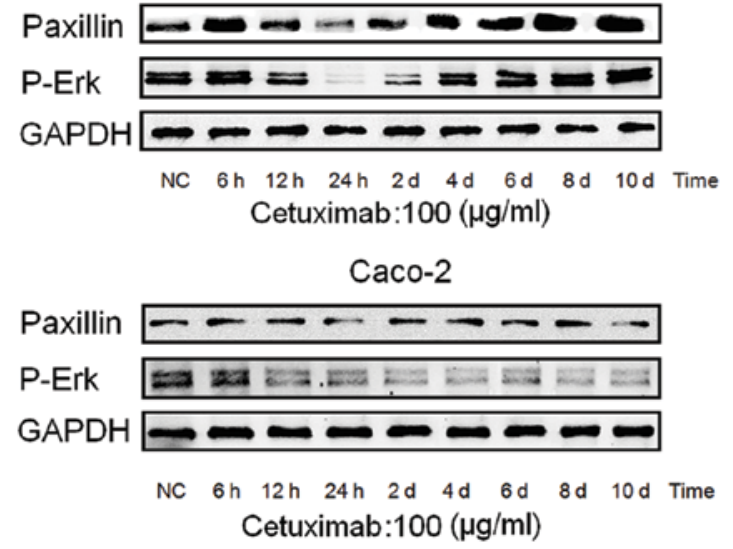

B

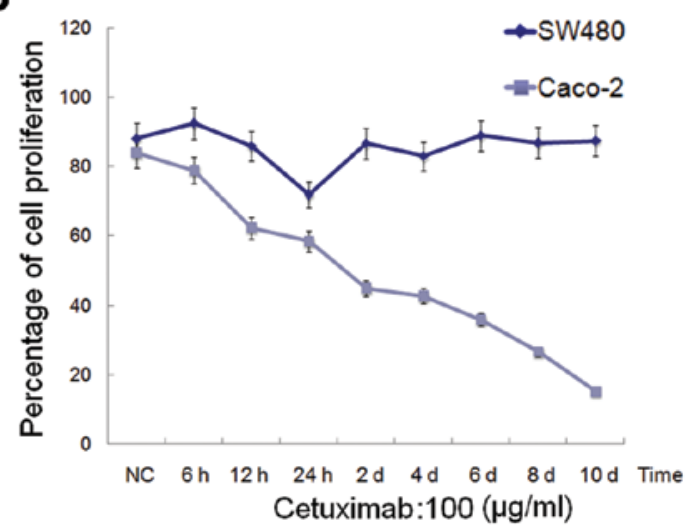

D

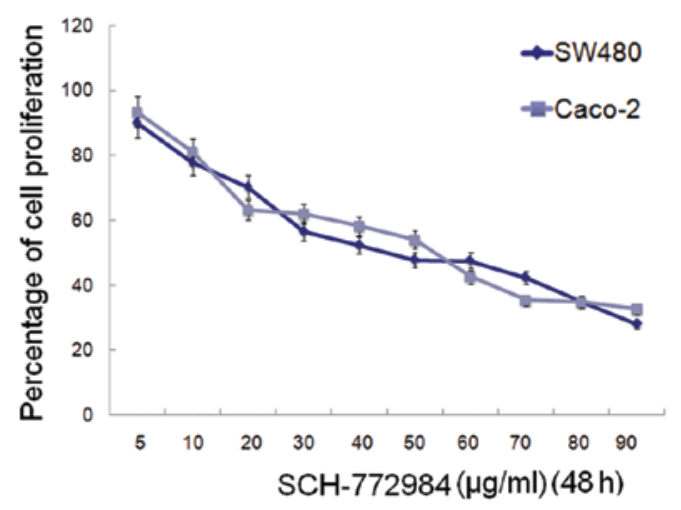

Figure 3. High expression level of paxillin may confer resistance to cetuximab in SW480 cells. (A and B) SW480 cells were not sensitive to cetuximab, and expressed a higher level of paxillin. Compared with the SW480 cells, Caco-2 cells were relatively sensitive to cetuximab and the $\mathrm{IC}_{50} \mathrm{value}$ was $\sim 189.3 \mathrm{mg} / \mathrm{ml}$. (C) The expression of paxillin in the SW480 cells following treatment with cetuximab declined at first, then increased gradually and finally stabilized, consistent with the proliferation curve in B. In contrast to SW480 cells, paxillin expression in the Caco-2 cells did not significant change. Moreover, the expression changes in p-Erk in both SW480 and Caco-2 cells were consistent with their proliferation rate. (D) Both SW480 and Caco-2 cells were sensitive to SCH-772984.

the colorectal adenocarcinoma samples than that in the normal colorectal mucosa samples $(68.2$ vs. $44.4 \%, \mathrm{P}<0.001$, Table I). The rate of recurrence in the colorectal patients was higher in the PXN-positive group (27.7\%) than that in the PXN-negative group (12.8\%, $\mathrm{P}=0.032$; Table I). PXN positivity was closely related to TNM stage $(86.4,74.6,61.5$ and $50.0 \%, \mathrm{P}=0.023$, Table I) and distant metastasis ( 88 vs. $64.2 \%, \mathrm{P}=0.014$; Table I).

High expression level of PXN confers resistance to cetuximab in SW480 cells. SW480 and Caco-2 cells were treated with increasing concentrations of cetuximab $(5-700 \mathrm{mg} / \mathrm{ml})$. The percentage of proliferation in the SW480 cells slightly increased at first and then slightly decreased (Fig. 3A). This showed that the SW480 cells were not sensitive to cetuximab. Compared with the SW480 cells, the Caco- 2 cells were relatively sensitive to cetuximab, and the $\mathrm{IC}_{50}$ value was $\sim 189.3 \mathrm{mg} / \mathrm{ml}$ (Fig. 3A).

To explore how PXN and p-Erk change with the treatment of cetuximab in SW480 and Caco-2 cells, the cells were treated for a period of time (from $6 \mathrm{~h}$ to 10 days) at a concentration of $100 \mathrm{mg} / \mathrm{ml}$ (Fig. 3B). As shown in Fig. 3C, the expression of PXN declined at first, and then increased gradually and stabilized in the SW480 cells, which was very consistent with the proliferation rate in Fig. 3B, the lowest point of which was at $24 \mathrm{~h}$. In contrast to the SW480 cells, PXN expression in the Caco-2 cells did not significantly change. These data demonstrated that the high expression level of PXN may be one of the reasons for the insensitivity to cetuximab of SW480 cells, which warrants further experiments for confirmation. Moreover, the expression changes in p-Erk in both the SW480 and Caco-2 cells (Fig. 3C) were consistent with the proliferation rates in Fig. 3B.

To clarify the effect of an Erk1/2 inhibitor on SW480 and Caco- 2 cells, the cells were treated with increasing concentrations of SCH-772984 (5-90 mg/ml) for $48 \mathrm{~h}$. SCH-772984 treatment of the SW480 and Caco-2 cells induced a dose-dependent inhibition of cell growth with an $\mathrm{IC}_{50}$ value of $\sim 47.5$ and $56.4 \mathrm{mg} / \mathrm{ml}$, respectively (Fig. 3D), which demonstrated that both SW480 and Caco-2 cells were sensitive to SCH-772984.

Inhibition of PXN expression by PXN-siRNA restores sensitivity to cetuximab in the SW480 cells. To determine whether PXN activation could be one of the reasons for cetuximab resistance in SW480 cells, a further investigation was carried out to ascertain whether reduction in PXN expression restores cetuximab sensitivity. First of all, we compared the effect of transfection between siRNA1 and siRNA2. As shown in Fig. 4A, transfection with siRNA1 for 72 h significantly 
A

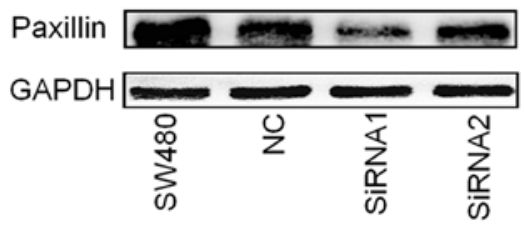

B
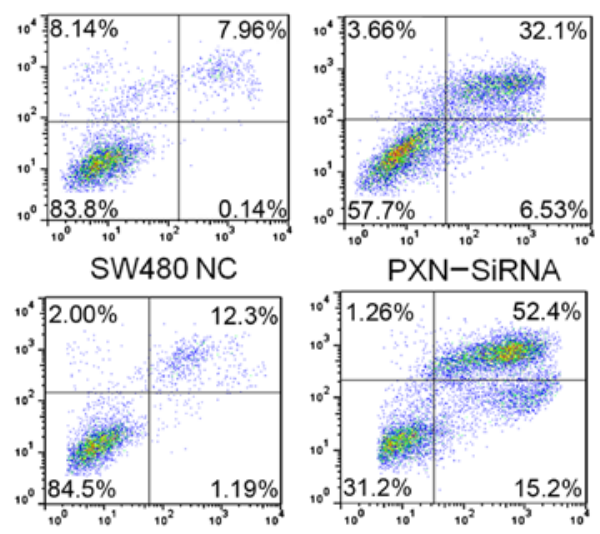

Cetuximab

C

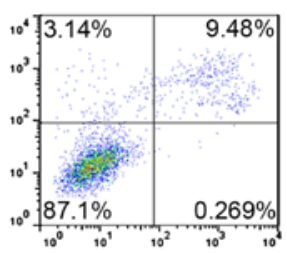

Caco-2 NC

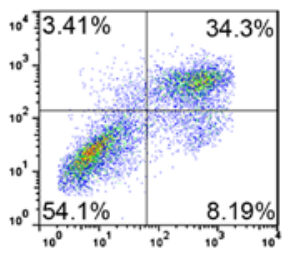

Cetuximab

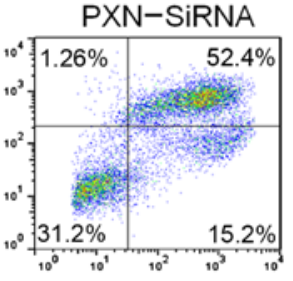

Cetuximab + PXN-SiRNA

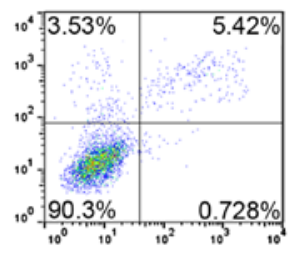

$\mathrm{PXN}-\mathrm{CDNA}$

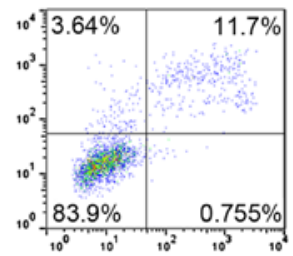

Cetuximab $+\mathrm{PXN}-\mathrm{cDNA}$
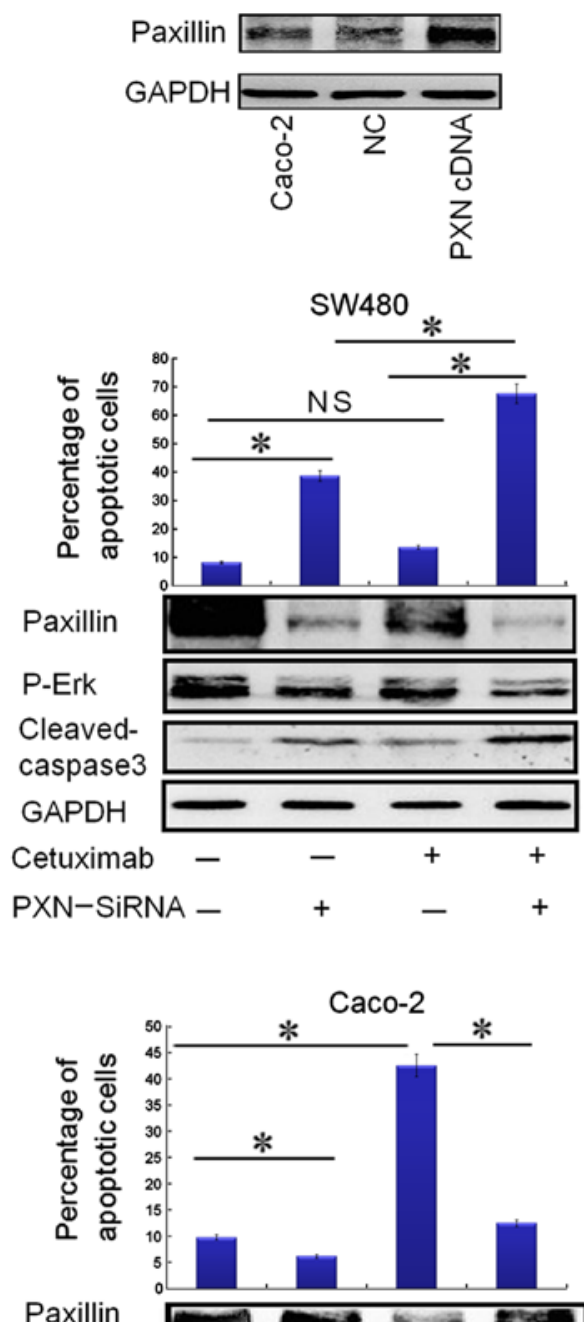

Paxillin

P-Erk

Cleavedcaspase 3

GAPDH

Cetuximab
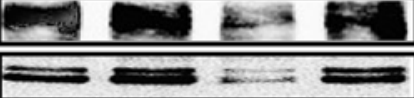

ए

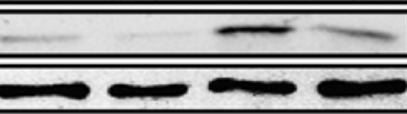

$\mathrm{PXN}-\mathrm{CDNA}-\quad+\quad-\quad+$

Figure 4. Downregulation of paxillin by PXN-siRNA significantly restores the sensitivity to cetuximab in SW480 cells and upregulation of paxillin expression by PXN-cDNA (PXN Human cDNA ORF Clone) reduces the sensitivity to cetuximab in the Caco-2 cells. (A) Transfection with siRNA1 (PXN-siRNA) for $72 \mathrm{~h}$ significantly reduced paxillin protein expression in the SW480 cells, and the expression level of paxillin increased significantly in the Caco-2 cells after transfection with PXN-cDNA for $72 \mathrm{~h}$. (B) Downregulation of paxillin by PXN-siRNA in combination with cetuximab obviously increased the apoptotic rate in the SW480 cells, illustrated by flowgram and histogram. Columns, means of three independent experiments. PXN-siRNA vs. negative control (NC) ("P $<0.001)$, cetuximab plus PXN-siRNA vs. PXN-siRNA ("P $<0.001)$, cetuximab plus PXN-siRNA vs. cetuximab ("P $<0.001)$, cetuximab vs. negative control (NC) (not significant NS, P $>0.05$ ). The expression of apoptotic protein cleaved caspase-3 was consistent with the data in the flowgram and histogram. Paxillin silencing also restored the ability of cetuximab to inhibit p-Erk activation in the SW480 cells, as shown by downregulation of p-Erk levels. (C) Upregulation of paxillin by PXN-cDNA clearly reduced the apoptotic rate in Caco-2 cells, illustrated by the flowgram and histogram. Columns: means of three independent experiments. Cetuximab vs. negative control (NC) ("P<0.001), PXN-cDNA vs. negative control (NC) ("P<0.05), PXN-cDNA plus cetuximab vs. cetuximab $($ ( $\mathrm{P}<0.005)$. Corresponding to the data, the expression of apoptotic protein cleaved caspase-3 was downregulated as the apoptotic rate declined. Paxillin overexpression also promoted the activation of p-Erk in Caco-2 cells, as shown by upregulation of p-Erk levels.

reduced PXN protein expression in the SW480 cells, and thus, siRNA1 (PXN-siRNA) was chosen to carry out the following transfection study.

Although single-agent cetuximab did not significantly affect SW480 apoptosis, cetuximab treatment in combination with PXN silencing showed a statistically significant apoptotic effect on SW480 cells (Fig. 4B). The expression of apoptotic protein cleaved caspase-3 was consistent with the apoptotic rate as shown in Fig. 4B. PXN silencing also inhibited p-Erk activation in the SW480 cells as shown by downregulation of p-Erk levels (Fig. 4B), which may be the mechanism through which SW480 cells regained sensitivity to cetuximab. 
A

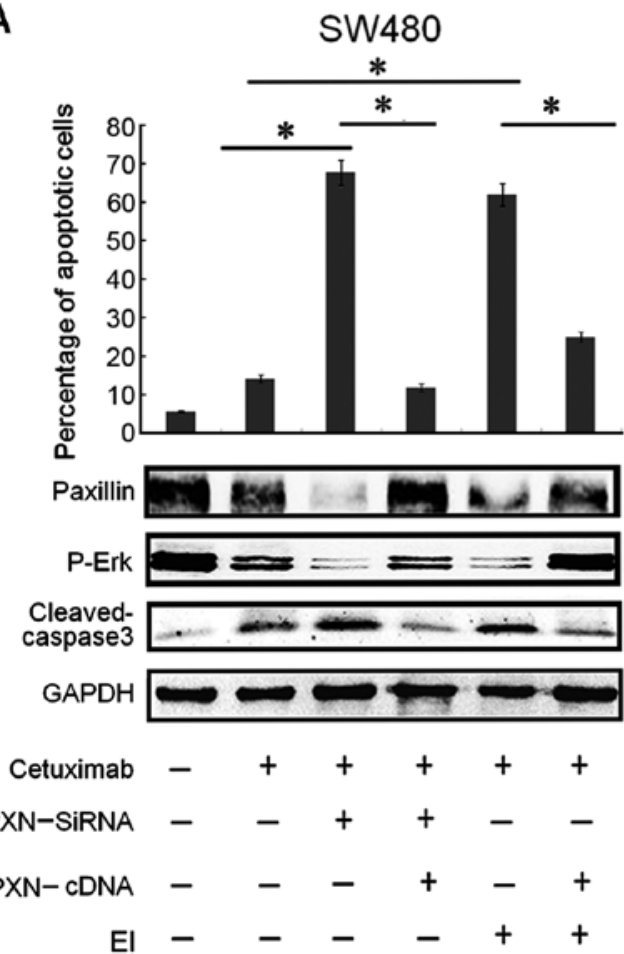

B
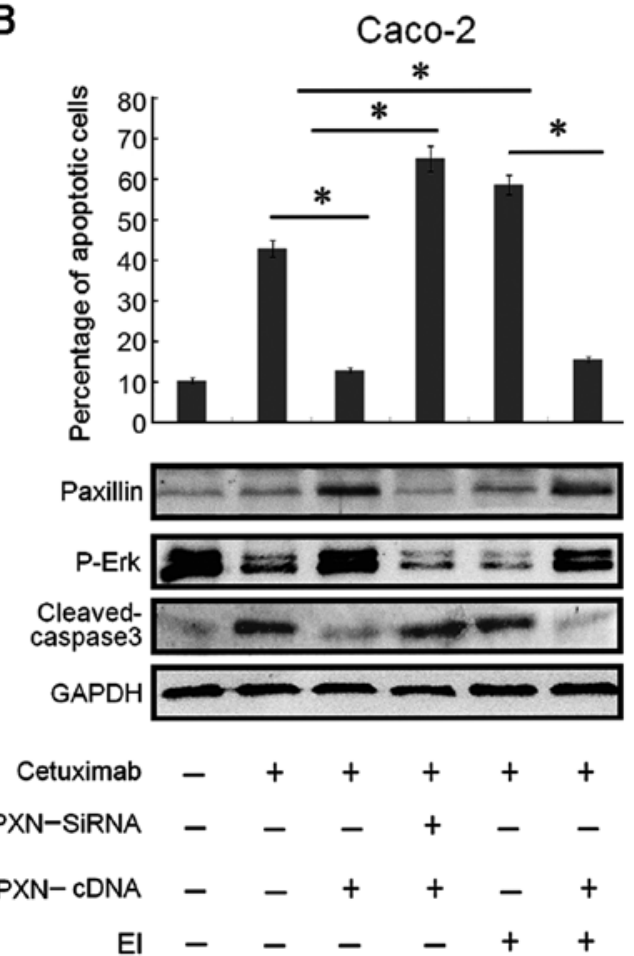

Figure 5. Paxillin overexpression regulates the resistance to cetuximab in a p-Erk-dependent pattern in SW480 and Caco-2 cells. (A) Both downregulation of paxillin and treatment with a selective Erk inhibitor (EI) drastically increased the apoptotic rate in SW480 cells, which were illustrated by histograms. Columns: means of three independent experiments. Cetuximab plus PXN-siRNA vs. cetuximab ( $\mathrm{P}<0.001)$, PXN-siRNA plus cetuximab vs. PXN-cDNA plus PXN-siRNA plus cetuximab ("P $<0.001)$, cetuximab plus EI vs. PXN-cDNA plus EI plus cetuximab ("P<0.005), cetuximab plus EI vs. cetuximab ("P $<0.001)$. The expression of apoptotic protein cleaved caspase-3 in the SW480 cells changed corresponding to its apoptotic rate. The expression of paxillin increased as expected after co-transfection, compared with transfection with PXN-siRNA alone. Activitation of paxillin also upregulated p-Erk levels. (B) Although Caco-2 cells were relatively sensitive to cetuximab, downregulation of paxillin and treatment with a selective Erk inhibitor (EI) increased the apoptotic rate in the Caco-2 cells, which was illustrated by histograms. Columns, means of three independent experiments. Cetuximab plus PXN-cDNA vs. cetuximab ( $\mathrm{P}<0.005)$, PXN-cDNA plus cetuximab vs. PXN-cDNA plus PXN-siRNA plus cetuximab (" $\mathrm{P}<0.001)$, cetuximab plus EI vs. cetuximab ("P $<0.001)$, cetuximab plus EI vs. PXN-cDNA plus EI plus cetuximab ("P<0.001). The expression of apoptotic protein cleaved caspase-3 corresponded to the apoptotic rate. The paxillin expression decreased as expected after co-transfection, compared with transfection with PXN-cDNA alone. Inhibition of paxillin expression also downregulated p-Erk levels.

Activation of PXN by PXN-cDNA (PXN Human cDNA ORF Clone) transfection reduces the sensitivity to cetuximab in the Caco-2 cells. To further evaluate whether PXN activation confers resistance to cetuximab in colorectal cancer cells, the Caco-2 cell line, confirmed to have low expression of PXN, was used. After transfection with PXN-cDNA for $72 \mathrm{~h}, \mathrm{PXN}$ protein expression was significantly increased in the Caco-2 cells (Fig. 4A). As illustrated in Fig. 4C, the overexpression of PXN effectively suppressed apoptosis in the Caco-2 cells with or without cetuximab treatment. Corresponding to the apoptotic data, the expression of apoptotic protein cleaved caspase-3 was downregulated with the decrease in the apoptotic rate (Fig. 4C). PXN overexpression also promoted the activation of $\mathrm{p}$-Erk in the Caco-2 cells as shown by upregulation of p-Erk levels (Fig. 4C). These data further demonstrated that $\mathrm{PXN}$ confers resistance to cetuximab and knockdown of PXN improves sensitivity to cetuximab in colorectal cancers by downregulating the expression of p-Erk.

PXN overexpression regulates resistance to cetuximab in a p-Erk-dependent pattern in the SW480 and Caco-2 cells. To further evaluate the role of PXN activation in cetuximab resistance, co-transfection with PXN-siRNA and PXN-cDNA in SW480 and Caco-2 cells was performed. Compared with transfection with PXN-siRNA alone, the apoptotic rate was greatly reduced after co-transfection with PXN-siRNA and PXN-cDNA for $72 \mathrm{~h}$ in the SW480 cells (Fig. 5A). The expression of apoptotic protein cleaved caspase-3 increased corresponding to the apoptotic rate (Fig. 5A). Moreover, the expression of PXN increased as expected after co-transfection, compared with transfection with PXN-siRNA alone and activation of PXN also upregulated p-Erk levels (Fig. 5A). Similarly, after co-transfection with PXN-siRNA and PXN-cDNA for $72 \mathrm{~h}$ in the Caco-2 cells, the apoptotic rate was greatly reduced, compared with transfection with PXN-cDNA alone (Fig. 5B). Not surprisingly, the expression of apoptotic protein cleaved caspase-3 increased, and activation of PXN and p-Erk was inhibited as shown by downregulation of p-Erk levels (Fig. 5B).

From the experiments above, we found that the expression levels of protein PXN and p-Erk were very synchronous. To explore the relationship between PXN and p-Erk and further evaluate the role of PXN activation, SW480 and Caco-2 cells were treated withcetuximab and SCH-772984, a selectiveErk1/2 inhibitor. SCH-772984 treatment of SW480 and Caco-2 cells induced a dose-dependent inhibition of cell growth with an $\mathrm{IC}_{50}$ value of $\sim 47.5$ and $56.4 \mathrm{mg} / \mathrm{ml}$, respectively (Fig. 3D). Although cetuximab treatment had little effect on cell growth in the SW480 cells, the combined treatment with SCH-772984 
restored the sensitivity of SW480 cells to cetuximab (Fig. 5A). Moreover, as illustrated in Fig. 5A, the percentage of apoptotic cells also obviously increased, compared with the group treated with cetuximab or SCH-772984 alone. Meanwhile, the expression levels of PXN and p-Erk were suppressed significantly in both cell lines (Fig. 5A and B). Based on the outcomes above, another test was performed to make the entire experiment more rigorous. After transfection with PXN-cDNA in the SW480 and Caco-2 cells for $24 \mathrm{~h}$, the cells were treated with cetuximab and SCH-772984 for another $48 \mathrm{~h}$. The results in Fig. 5A and B showed that the percentage of apoptotic cells decreased significantly, compared with the group that was treated only with cetuximab or SCH-772984. Correspondingly, the downregulation of cleaved caspase-3 and upregulation of both PXN and p-Erk expression levels are illustrated in Fig. 5A and B. In summary, knockdown of PXN restored or improved sensitivity to cetuximab by downregulating the expression level of p-Erk.

\section{Discussion}

Clarifying the mechanisms of colorectal cancer cell resistance to cetuximab is critical for the development of effective targeted therapies. In the past few years, extensive effort has been made to elucidate the mechanisms of cetuximab resistance. At present, it is generally acknowledged that specific gene mutations are responsible for resistance to anti-EGFR therapies in patients with colorectal cancer, including the KRAS, BRAF, NRAS, PI3KCA (exon 20) genes or inactivation of the PTEN phosphatase $(3,8,18)$. However, $\sim 25 \%$ of colorectal cancer patients with wild-type KRAS, BRAF, NRAS, PI3KCA and PTEN genes do not respond to EGFR inhibitors (3), which suggests there exists some other mechanism of resistance.

By analyzing the clinical data, it was demonstrated that the positive rate of paxillin (PXN) was much higher in the adenocarcinoma samples than in the normal mucosa samples (68.2 vs. $44.4 \%, \mathrm{P}<0.001)$. The rate of recurrence in the colorectal cancer patients was higher in the PXN-positive group than this rate in the PXN-negative group (27.7 vs. $12.8 \%$, $\mathrm{P}=0.032$ ). $\mathrm{PXN}$ presence was closely related with TNM stage $(86.4,74.6,61.5$ and $50.0 \%, \mathrm{P}=0.023)$ and distant metastasis ( 88 vs. $64.2 \%, \mathrm{P}=0.014$ ). Univariate analyses revealed that the 3- and 5-year survival rates of the PXN-negative group were higher (71.8 and 65.3\%) than those of the positive group (52.5 and 35.4\%, log-rank testing, $\mathrm{P}=0.004)$. Above all, PXN could be a risk factor for distant metastasis, recurrence and reduced survival time. However, PXN was not found to be an independent predictor due to the small number of samples and follow-up time was not long enough. Several studies have shown that PXN plays an important role in regulating tumor cell proliferation and mediating signal transduction of epidermal growth factor (EGF) $(2,10-12)$. Various scholars have also demonstrated that PXN leads to resistance to drugs by inhibiting the apoptosis of cancer cells in non-small cell lung cancer (17). In the present study, following treatment of cetuximab in SW480 and Caco-2 cells for a period of time, the expression of PXN in SW480 cells declined at first, and then gradually increased and finally stabilized, which was consistent with the proliferation rate. Different from SW480 cells, PXN expression in Caco-2 cells decreased gradually and the downward trend also corresponded to the proliferation curve. These data demonstrated that a high expression level of PXN may be one of the reasons for the insensitivity to cetuximab of SW480 cells in addition to the fact that the KRAS gene in these cells is mutant. It was hypothesized that high expression of PXN could be one reason for cetuximab resistance in metastatic colorectal cancer (mCRC) patients.

The SW480 cell line was chosen to conduct the first part of the experiment. It was reported that the expression of PXN in SW480 cells is very high and its KRAS gene is in a mutational status $(2,4)$. In the present study, we confirmed the high expression of PXN and that SW480 cells were not sensitive to cetuximab. The main reason for the resistance to cetuximab may be that the signaling pathway in the downstream of KRAS was not blocked although EGFR was inhibited by cetuximab treatment $(19,20)$. The high expression of PXN may also be one of the reasons. Our results showed that knockdown of PXN by PXN-siRNA transfection restored the sensitivity to cetuximab in the SW480 cells. As for the mechanisms, it is assumed that PXN plays a role in regulating the signaling pathway in the downstream of KRAS. As described in the Introduction, activation of the ERK signaling pathway requires the participation and mediation of PXN in prostate cancer and non-small cell lung cancer $(10,17)$. Moreover, Ishibe et al demonstrated that PXN serves as a scaffold for the organization and activation of the MAPK proteins Raf, Mek and Erk at focal adhesions (21). Ishibe et al also pointed out that Erk can either associate directly with PXN or interact via an intermediate protein (11). Several studies have demonstrated that Erk always gives a positive feedback on PXN and plays an important part in the activitation of PXN and interaction between FAK and PXN $(11,22,23)$. Based on these facts, we decided to examine the expression of p-Erk during this experiment. Following different treatments, the expression levels of p-Erk and PXN were always consistent. Moreover, the cells were treated with a selective Erk inhibitor, which resulted in downregulation of PXN and p-Erk, upregulation of apoptotic protein cleaved caspase-3 and an increase in the apoptotic rate. These results suggest that low expression of p-Erk could significantly increase the percentage of apoptotic cells and PXN may play a relevant role in regulating the expression of p-Erk. To further evaluate the role of PXN, upregulation of PXN expression was carried out in the Caco- 2 cell line since the expression of PXN in Caco-2 cells is low and its KRAS gene is wild-type (4). Thus, PXN-cDNA transfection was more effective. Upregulation of PXN by PXN-cDNA decreased the percentage of apoptotic cells and increased the expression levels of p-Erk and paxilllin in the Caco-2 cells. Treatment of Erk inhibitor was also carried out in Caco-2 cells, the results of which were similar with that in the SW480 cells.

The results of the present study suggest that PXN plays a relevant role in cetuximab resistance, and inhibition of PXN expression restores the sensitivity to cetuximab in colorectal cancer cells by downregulating p-Erk levels. In order to find more evidence to support the conclusion above, the co-transfection of PXN-cDNA and PXN-siRNA was performed in SW480 and Caco-2 cells. Compared with transfection with PXN-siRNA or PXN-cDNA alone, the same results were obtained following co-transfection in both the SW480 and Caco-2 cells, showing that overexpression of 
PXN could be one of the reasons for cetuximab resistance and downregulation of PXN plays an important role in improving the sensitivity to cetuximab by suppressing the activation of p-Erk in SW480 and Caco-2 cells. Moreover, the MAPK pathway was completely blocked when activation of p-Erk was inhibited. Knockdown of PXN in combination with cetuximab blocked the EGF pathway more completely, particularly when the genes located upstream of Erk were mutant. Besides the overexpression of PXN, there must exist other reasons for cetuximab resistance. In the past few years, researchers have focused on the relationship among apoptosis, drug resistance and autophagy in cancer cells. Various studies have demonstrated that increased induction of autophagy can become a mechanism of allowing tumor cells to survive the conditions of hypoxia, acidosis or chemotherapy, which results in a decrease in apoptosis and drug resistance $(24,25)$. There are also other studies indicating out that autophagy inhibitors in combination with chemotherapeutic agents may provide a premise for the treatment of colorectal cancer (26-28). Taken together, these results suggest that overexpression of PXN could be one of the reasons for cetuximab resistance, and knockdown of PXN plays an important role in improving sensitivity to cetuximab in colorectal cancer cells. Downregulation of the p-Erk level that results from the decreased expression of PXN blocks the EGF/MAPK pathway more completely. Therefore, knockdown of PXN represents a rational therapeutic strategy for increasing the sensitivity or overcoming cetuximab resistance in patients with colorectal cancer.

\section{References}

1. Troiani T, Martinelli E, Napolitano S, Vitagliano D, Ciuffreda LP, Costantino S, Morgillo F, Capasso A, Sforza V, Nappi A, et al: Increased TGF- $\alpha$ as a mechanism of acquired resistance to the anti-EGFR inhibitor cetuximab through EGFR-MET interaction and activation of MET signaling in colon cancer cells. Clin Cancer Res 19: 6751-6765, 2013.

2. Yin H, Zhang Q, Wang X, Li T, Wan Y, Liu Y and Zhu J: Role of paxillin in colorectal carcinoma and its relationship to clinicopathological features. Chin Med J 127: 423-429, 2014.

3. Bardelli A and Siena S: Molecular mechanisms of resistance to cetuximab and panitumumab in colorectal cancer. J Clin Oncol 28: 1254-1261, 2010.

4. Shigeta K, Hayashida T, Hoshino Y, Okabayashi K, Endo T, Ishii Y, Hasegawa $\mathrm{H}$ and Kitagawa Y: Expression of epidermal growth factor receptor detected by cetuximab indicates its efficacy to inhibit in vitro and in vivo proliferation of colorectal cancer cells. PLoS One 8: e66302, 2013.

5. Galizia G, Lieto E, De Vita F, Orditura M, Castellano P, Troiani T, Imperatore V and Ciardiello F: Cetuximab, a chimeric human mouse anti-epidermal growth factor receptor monoclonal antibody, in the treatment of human colorectal cancer. Oncogene 26: 3654-3660, 2007.

6. Van Cutsem E, Köhne CH, Láng I, Folprecht G, Nowacki MP, Cascinu S, Shchepotin I, Maurel J, Cunningham D, Tejpar S, et al: Cetuximab plus irinotecan, fluorouracil, and leucovorin as first-line treatment for metastatic colorectal cancer: Updated analysis of overall survival according to tumor KRAS and $B R A F$ mutation status. J Clin Oncol 29: 2011-2019, 2011.

7. Chen MC, Chiang FF and Wang HM: Cetuximab plus chemotherapy as first-line treatment for metastatic colorectal cancer: Effect of KRAS mutation on treatment efficacy in Taiwanese patients. Neoplasma 60: 561-567, 2013.

8. De Roock W, Claes B, Bernasconi D, De Schutter J, Biesmans B, Fountzilas G, Kalogeras KT, Kotoula V, Papamichael D, LaurentPuig P, et al: Effects of $K R A S, B R A F, N R A S$, and PIK3CA mutations on the efficacy of cetuximab plus chemotherapy in chemotherapy-refractory metastatic colorectal cancer: A retrospective consortium analysis. Lancet Oncol 11: 753-762, 2010.
9. Misale S, Yaeger R, Hobor S, Scala E, Janakiraman M, Liska D, Valtorta E, Schiavo R, Buscarino M, Siravegna G, et al: Emergence of KRAS mutations and acquired resistance to anti-EGFR therapy in colorectal cancer. Nature 486: 532-536, 2012.

10. Munshi N, Groopman JE, Gill PS and Ganju RK: c-Src mediates mitogenic signals and associates with cytoskeletal proteins upon vascular endothelial growth factor stimulation in Kaposi's sarcoma cells. J Immunol 164: 1169-1174, 2000.

11. Ishibe S, Joly D, Zhu X and Cantley LG: Phosphorylationdependent paxillin-ERK association mediates hepatocyte growth factor-stimulated epithelial morphogenesis. Mol Cell 12: $1275-1285,2003$.

12. Sen A, O'Malley K, Wang Z, Raj GV, Defranco DB and Hammes SR: Paxillin regulates androgen-and epidermal growth factor-induced MAPK signaling and cell proliferation in prostate cancer cells. J Biol Chem 285: 28787-28795, 2010.

13. Turner CE: Paxillin interactions. J Cell Sci 113: 4139-4140, 2000

14. Xiao LJ, Zhao EH, Zhao S, Zheng X, Zheng HC, Takano Y and Song HR: Paxillin expression is closely linked to the pathogenesis, progression and prognosis of gastric carcinomas. Oncol Lett 7: 189-194, 2014.

15. Jun Q, Zhiwei W, Lilin M, Jing K and Qichao N: Effects of paxillin on HCT-8 human colorectal cancer cells. Hepatogastroenterology 58: 1951-1955, 2011.

16. Chen DL, Wang DS, Wu WJ, Zeng ZL, Luo HY, Qiu MZ, Ren C, Zhang DS, Wang ZQ, Wang FH, et al: Overexpression of paxillin induced by miR-137 suppression promotes tumor progression and metastasis in colorectal cancer. Carcinogenesis 34: 803-811, 2013.

17. Wu DW, Wu TC, Wu JY, Cheng YW, Chen YC, Lee MC, Chen CY and Lee H: Phosphorylation of paxillin confers cisplatin resistance in non-small cell lung cancer via activating ERK-mediated Bcl-2 expression. Oncogene 33: 4385-4395, 2014.

18. Therkildsen C, Bergmann TK, Henrichsen-Schnack T, Ladelund $\mathrm{S}$ and Nilbert M: The predictive value of $K R A S, N R A S$, $B R A F, P I K 3 C A$ and PTEN for anti-EGFR treatment in metastatic colorectal cancer: A systematic review and meta-analysis. Acta Oncol 53: 852-864, 2014.

19. Brand TM, Iida M and Wheeler DL: Molecular mechanisms of resistance to the EGFR monoclonal antibody cetuximab. Cancer Biol Ther 11: 777-792, 2011.

20. Kumar SS, Price TJ, Mohyieldin O, Borg M, Townsend A and Hardingham JE: KRAS G13D mutation and sensitivity to cetuximab or panitumumab in a colorectal cancer cell line model. Gastrointest Cancer Res 7: 23-26, 2014.

21. Ishibe S, Joly D, Liu ZX and Cantley LG: Paxillin serves as an ERK-regulated scaffold for coordinating FAK and Rac activation in epithelial morphogenesis. Mol Cell 16: 257-267, 2004.

22. Güller MC, André J, Legrand A, Setterblad N, Mauviel A, Verrecchia F, Daniel F and Bernuau D: c-Fos accelerates hepatocyte conversion to a fibroblastoid phenotype through ERK-mediated upregulation of paxillin-Serine178 phosphorylation. Mol Carcinog 48: 532-544, 2009.

23. Teranishi S, Kimura K and Nishida T: Role of formation of an ERK-FAK-paxillin complex in migration of human corneal epithelial cells during wound closure in vitro. Invest Ophthalmol Vis Sci 50: 5646-5652, 2009.

24. Çoker-Gürkan A, Arisan ED, Obakan P and Palavan-Unsal N: Lack of functional p53 renders DENSpm-induced autophagy and apoptosis in time dependent manner in colon cancer cells. Amino Acids 47: 87-100, 2015.

25. Sui X, Kong N, Wang X, Fang Y, Hu X, Xu Y, Chen W, Wang K, Li D, Jin W, et al: JNK confers 5-fluorouracil resistance in p53-deficient and mutant p53-expressing colon cancer cells by inducing survival autophagy. Sci Rep 4: 4694, 2014.

26. Li J, Hou N, Faried A, Tsutsumi S, Takeuchi T and Kuwano H: Inhibition of autophagy by 3-MA enhances the effect of 5-FU-induced apoptosis in colon cancer cells. Ann Surg Oncol 16: 761-771, 2009.

27. Nishikawa T, Tsuno NH, Okaji Y, Shuno Y, Sasaki K, Hongo K, Sunami E, Kitayama J, Takahashi K and Nagawa H: Inhibition of autophagy potentiates sulforaphane-induced apoptosis in human colon cancer cells. Ann Surg Oncol 17: 592-602, 2010.

28. Zhai H, Song B, Xu X, Zhu W and Ju J: Inhibition of autophagy and tumor growth in colon cancer by miR-502. Oncogene 32: 1570-1579, 2013 